\title{
Technique for Detection and Isolation of Jamming Attack for AODV Routing Protocol in MANET
}

\author{
Aditi $^{1}$ and Joy Karan Singh ${ }^{2}$ \\ ${ }^{1}$ M.tech student, Department of CSE CT Institute of Technology and Research \\ Maqsudan, Jalandhar \\ ${ }^{2}$ Assistant Professor, Department of ECE CT Institute of Technology and \\ Research Maqsudan, Jalandhar \\ ${ }^{1}$ sweetgagan608@gmail.com, ${ }^{2}$ joysachar@gmail.com
}

\begin{abstract}
The mobile ad-hoc network is the self configuring type of network in which mobile nodes can change its location any time. Due to its decentralized nature, much type of active and passive attacks is possible in the network. Jamming is the active type of attack which degrades network performance in terms of throughput, Packetloss, Retransmission attempts, Delay and PDR. In this work, novel technique is been proposed which will detect and isolate malicious nodes from the network. The proposed technique will be based on monitor mode and threshold. The simulation is been performed in NS2 and it is been analyzed that proposed technique performs better in terms of throughput, Packetloss, Retransmission attempts, Delay and PDR.
\end{abstract}

Keywords: MANET, AODV, Attacks, Jamming, QoS parameters

\section{Introduction}

The communication between computers with the help of standard network protocols is known as wireless networking technology. The communication is done without using any external cables in the network and the transmission is done through radio waves at the physical level. This network is also known as Wi-Fi or WLAN. The communication between two devices in this network can be done through radio frequency. The IEEE standard for wireless network is 802.11. There are two types of Wireless Operating modes:

a) Infrastructure Mode

b) Ad-hoc Mode or Infrastructure less Mode

Here, the communication in the infrastructure based network takes place between the wireless nodes and the access points and no direct communication is done between the wireless nodes. The medium access is handled by the access point and this access point acts as a bridge in between the wireless and wired networks. Fixed numbers of base stations are used in this network and if any node goes out of range of a particular base station, another base station comes in the range. Cellular networks are an example of the infrastructure based networks. In this network the infrastructure is centralized based and the controlling activity is done by the controller such as a router. On the contrary, the infrastructure less network does not require ant parameters for design and working. The communication with other nodes in this network can be done directly and there is no access point needed for controlling the medium access. All the nodes available in this network act as routers and are capable of moving. These nodes can also be connected dynamically in an arbitrary manner [6].

MANET (mobile ad hoc network) is a robust infrastructure less wireless network which is formed either by all mobile nodes or by both fixed as well as mobile nodes. Arbitrary topology is formed by connecting nodes randomly amongst each other. The 
nodes can act either as routers or as hosts. The nodes have the property of selfconfiguration which can be helpful in providing communication in areas where it is difficult to communicate. These are areas mainly which are disaster prone or emergency search and rescue operations in which the network connection is needed on urgent basis. There is need of both static as well as dynamic routing protocols in MANET. In an ad hoc network there is no centralized infrastructure available which results in imposing great challenge to the functionality of the network. To avoid this, MANETs are proposed which have the property of accepting and routing traffic from the intermediate nodes towards the destination. The nodes cannot act both as the routers as well as hosts. Energy constraint occurs in the network if there is frequent breakage of connection and re-connecting found in the network. The routing protocols that are used for communication play a very important role in this network. This is due to the fact that there is a huge impact on the complete network due to these routing protocols.

In this paper, we esteem a particular category of DoS attacks called Jamming. In actual fact, the mobile host in mobile ad hoc networks is a part of wireless medium. Thus, the radio signals can be jammed or interfered, which make the message to be amoral or missed. If the attacker has a strong transmitter, a signal can be launched that will be strong enough to conquer the directed signals and distort communications. There are several attack schemes that a jammer can do in order to interfere with other wireless communications. In proposed work we implemented ad-hoc on demand distance vector routing protocol on wireless sensor network because it select the shortest path for transferring data source to destination. But it cannot able to isolate jamming effectively. To increase the performance of the network and enhance the performance of AODV other technique is used Watch dog technique. Watchdog is a monitoring technique [3] which detects the misbehaving nodes in the network. . In this work Watchdog algorithm is implemented on AODV to detect the selfish node form the network. So, we implemented, analysis and compare the result of Watchdog technique with Adhoc- On Demand Distance Vector Routing Protocol. Watchdog algorithm in previous work implemented on mobile Adhoc network for avoiding jamming. We implemented Watchdog algorithm on AODV then analyze and compare the results with AODV jamming. Enhanced Watchdog technique is my proposed work which having increase the performance of network and AODV as well as decrease the chances of jamming. Lastly, we would like to analyze and compare the results of proposed technique Enhanced Watchdog technique with exiting Watchdog technique and AODV. By proposed Watchdog technique with other two I want to observe the difference between the performances of enhanced Watchdog technique, exiting Watchdog technique and AODV.

\section{Attacks in MANET}

Providing better security solutions to the wireless ad-hoc network is a major challenge in MANETs. For this purpose, it is important to study all the attacks that are possible to occur while there is data transmission occurring in the network. Numerous types of attacks are found in MANET. Basically the classification of attacks is done in two parts:

(i) Active attack: Active attacks are of two types, internal attacks and external attacks. These types of attacks affect the performance of the network. The tasks performed are also affected as the modified data could also be sent which might be a false message.

- Internal attacks: These types of attacks are found within the network. The attacker nodes are found within the network and these nodes take unauthorized access. They behave as normal nodes and thus disrupt the network on authentication base. The traffic in between the other nodes is also analyzed by these nodes and they begin to involve in various other activities of the network.

- External attacks: These types of attacks are made by the attacker nodes found outside the network. These nodes are not present within the network and create 
authentication issues from the outside. The examples of these types of attacks are: jamming, modification and message reply.

(ii) Passive attacks: Passive attacks are that kind of attacks which are difficult to find on the network and does not disturb the network task, performance and actions. The most common example of passive attacks is traffic analysis and traffic monitoring.

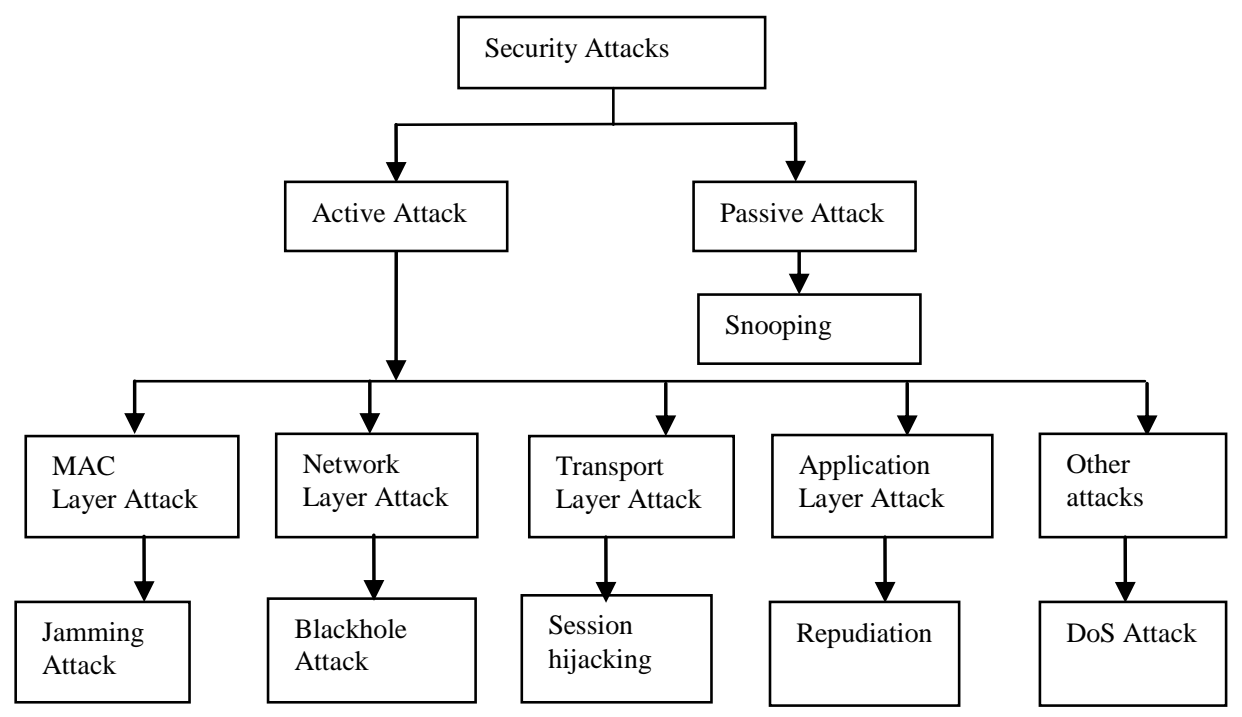

Figure 1. Security Attacks for Different Layers

Jamming Attack: The jammer is an entity with the aim of attempting to involve in the sending and receiving of data within the wireless communications of network. For blocking the legal traffic of the wireless channel, the jammer continuously emits RF signals. The jamming attacks have common properties which involve the usage of MAC protocols for their interactions [2]. A ratio of the number of packets sent out by any justifiable traffic source to the number of packets to be sent by the MAC layer is taken. This attack has a number of sources instead of just one source. These sources send the rough packets to the transmission channels and to the jammed channels as well. This results in packet loss which further decreases the efficiency and reliability of the system. The problems such as the unavailability of free channel, delay in transmission and new packet drops due to the absence of buffer space are seen.

The motive of jamming attack is to fill up the communication channel with purposeless signals, due to which verified or permissible user cannot use it. Jamming slowly down the receiving and sending of messages at the destination. It is very difficult to prevent and find out the jamming attacks but still some detection algorithms are struggling to prevent the prospects of jamming attack. Another motive of Jammers is to conceal themselves from the detection algorithms so that they can begin with jamming of some particular region. [13].

- Constant Jammer: A constant jammer is the signal alternator that does not obey any MAC protocol and it continuously released radio signal that represents random bits.

- Deceptive Jammer: They dispatch semi-valid packets. This means that the payload is bootless but the packet header is sustainable.

- Random Jammer: Substitutes between sleeping and jamming the channel. In the first modus the jammer jams for a casual period of time (it can behaves like a constant jammer or as a deceptive jammer), and in the second modus (the sleeping mode) the jammer spins its transmitters off for a different random period of time [6]. The energy efficiency is regulates as the ratio of the length of the jamming period upon the length of the sleeping period. 
- Reactive Jammer: A reactive jammer attempts not to misspend resources by only jamming when it recognize that somebody is transmitting. Its object is not the sender but the receiver, taxing to input as much noise as possible in the packet to improve as many bits as possible given that only a small amount of power is required to modify sufficient bits so that when a checksum is execute over that packet at the receiver it will be categorized as not valid and therefore discarded [6].

\section{Related Work}

Tang et al. [2010] proposed efficient authentication mechanisms for low-power devices. In the proposed scheme the mobile station only need to pass one packet for mutual authentication. They used the elliptic-curve-crypto system based trust delegation Mechanism to generated group pass code for mobile station authentication. The authentication mechanism is helpful in preventing networks from various active and passive attacks. The authentication of devices is done through the exchange of one packet while visiting the base station. When compared to the other authentication methods proposed, this method involves less computations and message exchange [1].

Goyal et al. [2011] discussed about the Mobile ad-hoc networks which are now used in various fields for evolving the research and development of wireless networks. There are various challenges faced by MANET which are to be resolved by enhancing the special properties of MANET. In this paper, the features, categories as well as vulnerabilities of MANET are mentioned for getting detailed view about these networks. Towards the end, the emerging applications as well as the future enhancements to be proposed in MANET are also mentioned [4].

Liu et al. [2012] proposed a novel two-phase jamming detection method for sensor networks. In first phase, some signs of jamming are identified speedily. When signs are found then second phase of detection is applied. In this technique we don't requires any extra communication or hardware [12].

Babar et al. [2013] represented the game theoretic model of the jamming attack. This paper suggested a game theory based detection technique which is utilized to detect all kinds of jamming attack. This method provides better performance in words of delay, energy and throughput also [13].

S. T. et al. [2014] represented a profile based technique which is utilized to detect and suspend the flooding attack on MANET with the help Adhoc on Demand Distance Vector (AODV) routing protocol. In this technique every single node has a profile value. These profile values are put on the base of behavior of MANET. Whenever the node attempts to overreach the fixed threshold value, the attack will be identified and isolated. The key benefit of this technique is that threshold value is not defined; it is based on the average request permitted in the network which changed with the number of request in the network [15].

\section{Proposed Methodology}

A partial DoS attack which is triggered by the malicious nodes in the network is known as jamming packet. To remove the jamming attacks from the network, various techniques have been proposed. The affects of jamming attack are that the throughput of the network gets reduced as well as the delay is increased at a steady rate. In this work, the jamming attack of AODV protocol is detected and isolated. The route is established between source and destination on the basis of hop counts and sequence numbers. The malicious node exists in the route which is act as source or multiple sources. The malicious node will be responsible for triggering the jamming attack. The proposed methodology will detect the malicious node and isolate, it from the network. The throughput of the network is of great concern in this methodology. The nodes go to the monitor mode once the threshold of the network degrades to certain threshold value. This 
helps in detection of the malicious node. In this work, the issue of dropped packet is resolved through its detection and redirection towards the source using the monitoring nodes. From the source side, the ICMP packets that are generated flood in the network. These packets further act as the monitor nodes. The monitoring nodes detect the malicious node which is not further sent to the destination. When the malicious node is detected by the nodes, the reply is sent to the source node. The path is isolated to the source which stops forwarding more packets further.

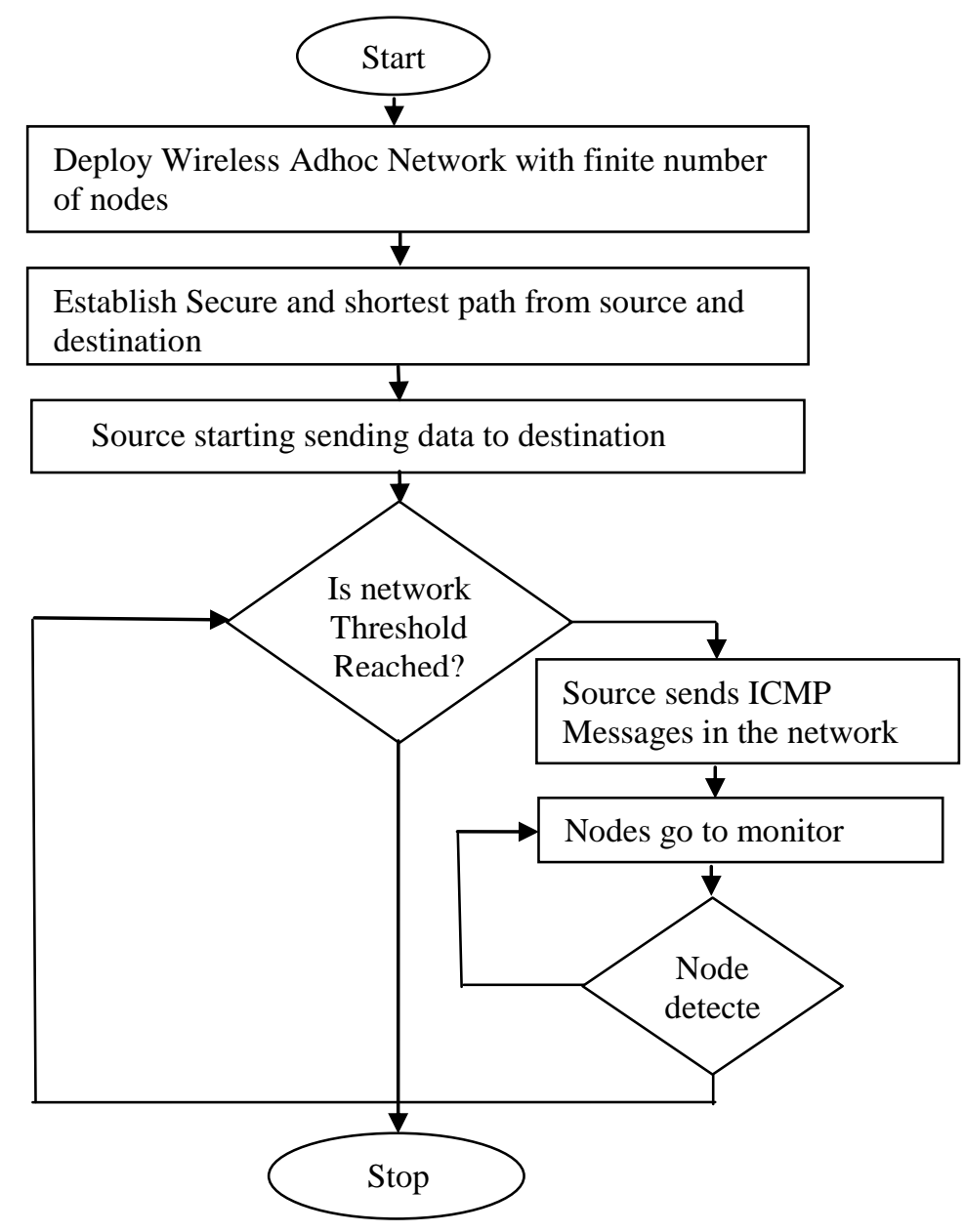

Figure 2. Flowchart of Proposed Technique

\section{Experimental Results}

\subsection{Delay}

Delay by the sensor nodes in the network is one of the essential metrics of routing protocol. Comparison graph of delay is shown in figure 3, which shows that delay by Enhanced Watchdog algorithm is low as compare to AODV jamming and exiting watchdog technique. Because Enhanced Watchdog technique isolates jamming attack by selecting path according to prediction value and provide alternative path. Enhanced Watchdog algorithm is effectively work to isolate the jamming attack that can be observed from the graphical result shown in figure 3. 


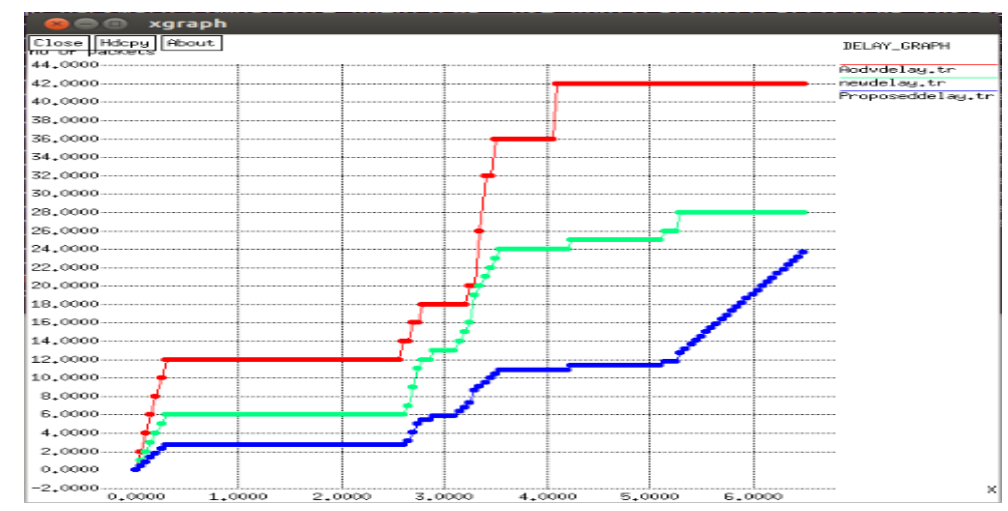

Figure 3. Delay Graph

\subsection{Throughput}

The throughput represents the ratio of numbers of data packets sent by the source node to the number of data packets received by the destination. Comparison graph of throughput is shown in figure 4, which shows that throughput of Enhanced Watchdog algorithm is high as compare to AODV jamming and exiting jamming algorithm. Because Enhanced Watchdog technique isolate jamming attack by selecting path according to prediction value and provide alternative path and also when the selected path gets jammed. Enhanced Watchdog algorithm is effectively work to isolate the jamming attack that can be observed from the graphical result shown in figure.

$$
\text { Throughput }=\quad \frac{\text { No. of data packets sent }}{\text { No. of data packets received }}
$$

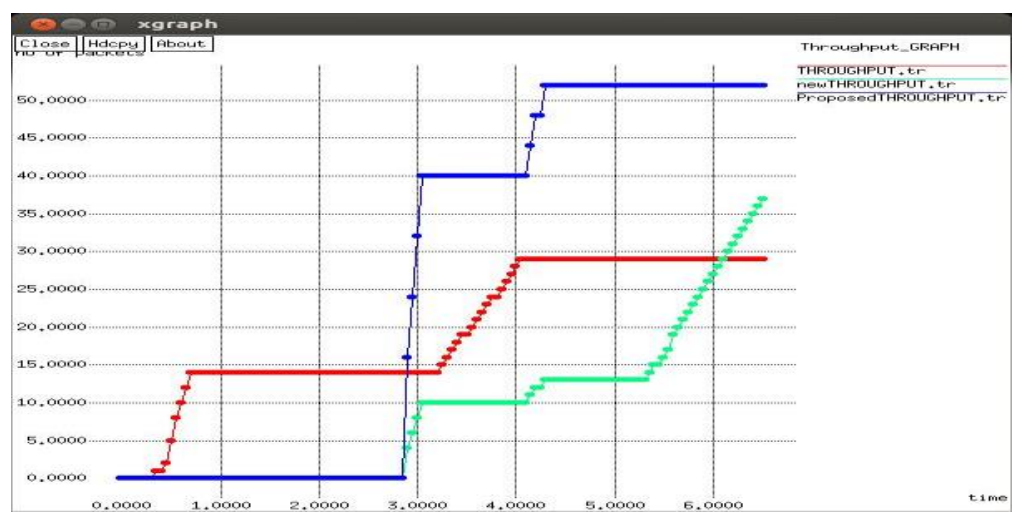

Figure 4. Throughput Graph

\subsection{Packet Delivery Ratio}

The packet delivery ratio is the ratio of total number of packets received at destination node to that of total number of packets sent by the source node. Comparison graph of Packet delivery ratio is shown in figure 5, which shows that packet delivery ratio of Enhanced Watchdog algorithm, is high as compare to AODV jamming and exiting watchdog technique. Because Enhanced Watchdog technique isolate jamming attack by selecting path according to prediction value and provide alternative path and also when the selected path gets jammed. Enhanced Watchdog algorithm is effectively work for isolate the jamming attack that can be observe from the graphical result shown in figure 5 . 
Packet delivery ratio $=\frac{\text { Total no. of packets receive }}{\text { Total no. of packets sent }}$

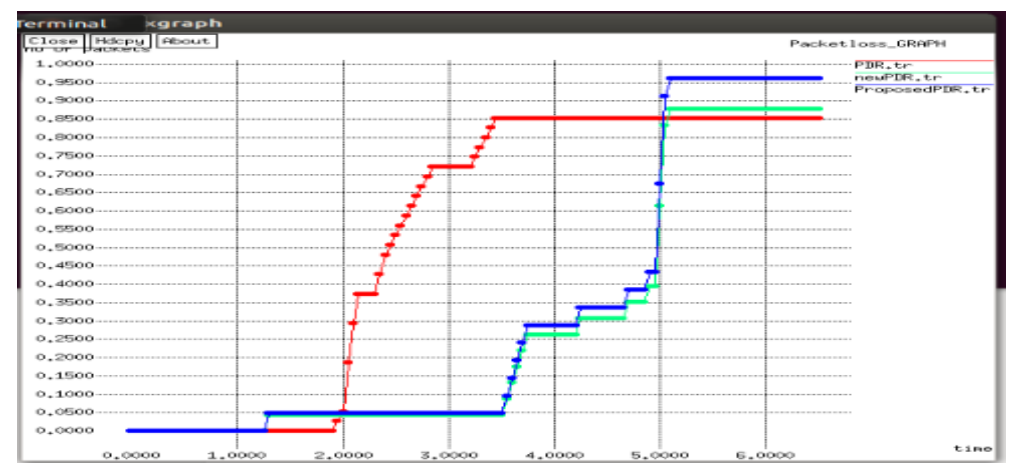

Figure 5. PDR Graph

\subsection{Packet Loss}

Packet loss is defined as total number of packets dropped in the network. Comparison graph of Packet loss is shown in figure 6, which shows that packet-loss of Enhanced Watchdog algorithm, is very low as compare to AODV jamming and exiting watchdog technique. Because Enhanced Watchdog technique isolate jamming attack by selecting path according to prediction value and provide alternative path and also when the selected path gets jammed. Enhanced Watchdog algorithm is effectively work for isolate the jamming attack that can be observed from the graphical result shown in figure 6 .

Packet loss $=$ Total number of packet send - Total number of packet received

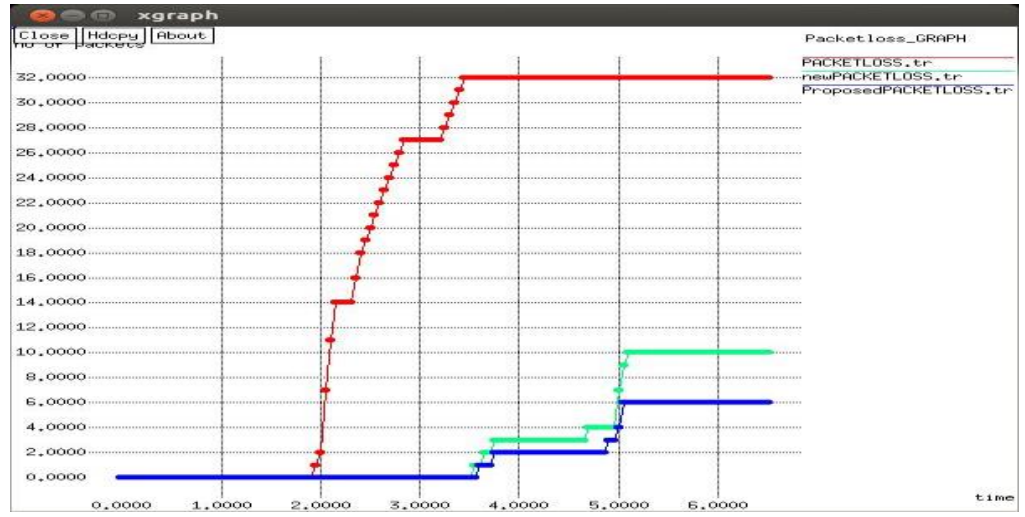

Figure 6. Packetloss Graph

\subsection{Retransmission Attempts}

Retransmission attempts happened in network only when the delivery of packet is dropped or lost without reaching to the destination nodes. Comparison graph of retransmission attempts is shown in figure 7 , which shows that retransmission of Enhanced Watchdog algorithm, is very low as compare to AODV jamming and exiting watchdog technique. Because Enhanced Watchdog technique isolate jamming attack by selecting path according to prediction value and provide alternative path and also when the selected path gets jammed. Enhanced Watchdog algorithm is effectively work for isolate the jamming attack that can be observed from the graphical result shown in figure 7. 


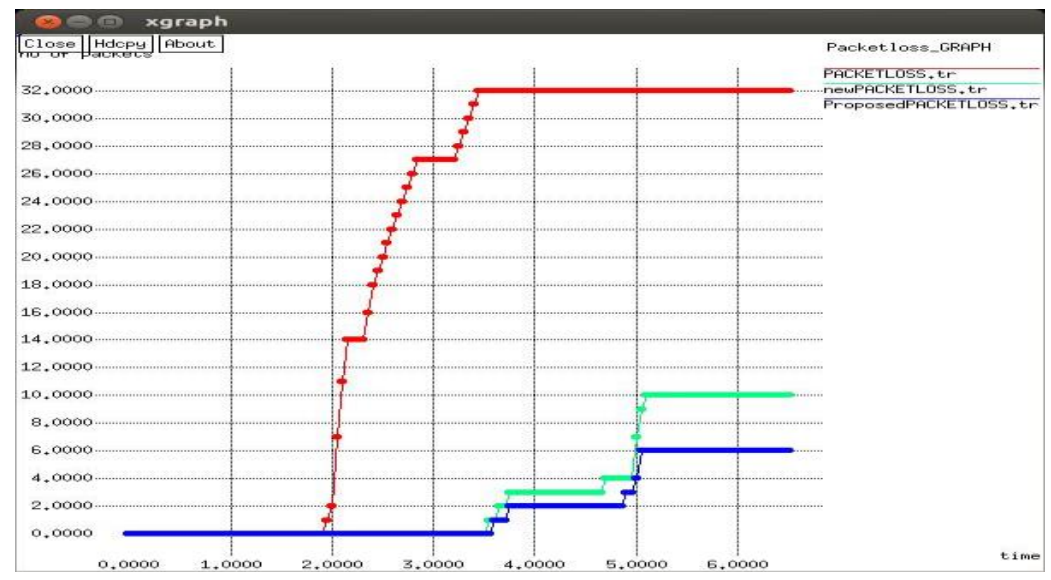

Figure 7. Retransmission Attempts Graph

\section{Result Analysis}

The result analysis of AODV jamming, Watchdog technique and Enhanced Watchdog technique are shown in this section with the help of the simulation outputs with five performance metrics. The aim of this comparative study of AODV jamming, Watchdog technique and Enhanced Watchdog technique is to analyze the performance of AODV protocol under enhanced watchdog technique. Enhanced Watchdog technique in our simulation experiments presents overall the best performance.

Table 1. Comparison of Technique

\begin{tabular}{|l|l|l|l|}
\hline $\begin{array}{l}\text { Performance } \\
\text { Metric }\end{array}$ & $\begin{array}{l}\text { AODV Jamming } \\
\text { (packet/Sec) }\end{array}$ & $\begin{array}{l}\text { Old algorithm } \\
\text { (packet/Sec) }\end{array}$ & $\begin{array}{l}\text { New Algorithm } \\
\text { (packet/Sec) }\end{array}$ \\
\hline Delay & 42 & 28 & 24 \\
\hline Packetloss & 25 & 9 & 6 \\
\hline Throughput & 29 & 37 & 53 \\
\hline PDR & 0.85 & 0.88 & 0.96 \\
\hline $\begin{array}{l}\text { Retransmission } \\
\text { attempts }\end{array}$ & 100 & 44 & 33 \\
\hline
\end{tabular}

\section{Conclusion}

Wireless Sensor Networks are commanly used in various fields for data monitoring purposes. They are helpful in mainly industrial, civilian and scientific applications. So it is important to detect jamming attack quickly because this attack seed DDoS on WSN. This paper recommends an efficient procedure for detection of these attacks. In this paper three types of simulation implemented. In first simulation results are carried with jamming attack on AODV, in second simulation results are carried with Watchdog technique on AODV, and in the end enhanced watchdog technique on AODV. In this procedure monitor mode is used to isolate the harmful path. To isolate the attack the source deluge (flood) ICMP packets in the network. Nodes which collect ICMP packets go to the monitor mode. One node which is neighbor to the harmful node detects 
malicious node and send message to source node to isolate the path. After this source node select the path according to the predictions value. Now source node isolate the path and the other path will be select for the communication on the basis of prediction value.

Enhanced Watchdog technique isolate jamming attack by selecting path according to prediction value and provide alternative path also when selected path gets jammed. Enhanced Watchdog technique is an Enhanced version of Watchdog algorithm due to which the results is better than individual result of two techniques. It means by using this enhanced technique the performance of network and AODV increased. The scheme has been assessed using the simulator NS-2. The results of our implementation present superior impact to overcome the jamming attack. This method noteworthy refins system performance and we find that the method is efficient because it detect jamming attack with less retransmission attempts, less delay, more throughput, less packet loss and more packet delivery ratio.

\section{Acknowledgment}

The authors would like to acknowledge the Department of Computer Science Engineering, CT Institute of Technology \& Research Jalandhar, India for the facilities provided during this research.

\section{References}

[1] Caimu Tang ,Dapeng Oilver,“ An Efficient Mobile Authentication Scheme for Wireless Networks”, IEEE, vol 7, issue 4, (2010), pp. 1408 - 1416.

[2] Jacek Cicho, Rafał Kapelko, Jakub Lemiesz, and Marcin Zawada "On Alarm Protocol in Wireless Sensor Networks", 9th International Conference, volume 6288, ISSN: 0302-9743, (2010), pp 43-52.

[3] Dempsey T., Sahin G., and Morton Y., "Passive and Active Analysis in DSR-Based Ad Hoc Networks," Ad Hoc Networks. Springer Berlin Heidelberg , pp. 623-638 , 2010.

[4] Priyanka Goyal, Vintra Parmar and Rahul Rishi "“ MANET: Vulnerabilities, Challenges, Attacks, Application”, IJCEM International Journal of Computational Engineering \& Management, Vol. 11, ISSN (Online): 2230-7893 (2011), pp. 345-358.

[5] Chen T., and Kuan W., "A Robust Mutual Authentication Protocol for Wireless Sensor Networks"ETRI Journal, Volume 32, Number 5, October 2010.

[6] Cicho J., Kapelko R., Lemiesz J., and Zawada M., "On Alarm Protocol in Wireless Sensor Networks", IEEE, 2010.

[7] Şen S., Clark J., and Tapiador j., "Security Threats in Mobile Ad Hoc Networks", IEEE, 2010.

[8] Defrawy K., and Tsudik G., "ALARM: Anonymous Location-Aided Routing in Suspicious MANETs", IEEE, Vol. 10, No. 9, September 2011.

[9] Vinit Garg, Manoj Kr.Shukla, Tanupriya Choudhury, Charu Gupta, “Advance Survey of Mobile Ad-Hoc Network,” IJCST Vol. 2, Issue 4, (2011), ISSN: 2229-4333.

[10] Humayun Bakht, "Survey of Routing Protocols for Mobile Ad-hoc Network”, International Journal of Information and Communication Technology Research, Volume 1 No. 6, (2011), ISSN-2223-4985.

[11] Donggang L., Raymer J., and Fox A., "Efficient and timely jamming detection in wireless sensor networks" Mobile Adhoc and Sensor Systems (MASS), 2012 IEEE 9th International Conference on IEEE, 2012.

[12] D. Liu, J. Raymer, A. Fox "Efficient and Timely Jamming Detection in Wireless Sensor Networks" in 9th International Conference on Mobile Adhoc and Sensor Systems MASS, page 335-343. IEEE Computer Society, December 2012.

[13] S. D. Babar, N. R. Prasad, R. Prasad "Game Theoretic Modelling of WSN Jamming Attack and Detection Mechanism" Published in Wireless Personal Multimedia Communications (WPMC), 2013.

[14] Rajakumar P., Prasanna T., and Pitchaikkannu A. "Security attacks and detection schemes in MANET," Electronics and Communication Systems (ICECS), 2014 International Conference on IEEE, 2014.

[15] Sathish. T, Sasikala. E" Dynamic Profile Based Technique to Detect Flooding Attack in MANET" in International Journal of Innovative Research in Computer and Communication Engineering Vol.2, Special Issue 1, March 2014.

[16] Kapur R., and Khatri S., "Analysis of attacks on routing protocols in MANETs," Computer Engineering and Applications (ICACEA), 2015 International Conference on Advances in IEEE, pp.791-798, 2015.

[17] Khan M., Jadoon Q., and Khan M., "A Comparative Performance Analysis of MANET Routing Protocols under Security Attacks," Mobile and Wireless Technology 2015, Springer Berlin Heidelberg, pp.137-145, 2015. 
International Journal of Advanced Science and Technology Vol.100 (2017) 